\title{
Metastatic Insulinoma: Current Molecular and Cytotoxic Therapeutic Approaches for Metastatic Well-Differentiated panNETs
}

\author{
Iulia Giuroiu, MD, and Diane Reidy-Lagunes, $\mathrm{MD}, \mathrm{MS}^{\mathrm{b}}$
}

\begin{abstract}
Pancreatic neuroendocrine tumors (panNETs) are a type of neuroendocrine tumor with 5-year overall survival rates of approximately $50 \%$ when metastasis is present at diagnosis. Tumor grade, as defined by Ki-67 proliferation index, influences overall survival, with low-grade tumors portending a better outcome than intermediate- and highgrade tumors. This case report follows the clinical course and management of a patient with an insulin-secreting metastatic panNET who died 10 years after diagnosis after a treatment course with regional therapy and multiple forms of cytotoxic and molecularly targeted agents. This report presents the various treatment options available for patients with insulin-secreting metastatic panNETs. (J Natl Compr Canc Netw 2015;13:139-144)
\end{abstract}

\section{NCCN: Continuing Education}

\section{Accreditation Statement}

This activity has been designated to meet the educational needs of physicians and nurses involved in the management of patients with cancer. There is no fee for this article. No commercial support was received for this article. The National Comprehensive Cancer Network (NCCN) is accredited by the ACCME to provide continuing medical education for physicians.

NCCN designates this journal-based CME activity for a maximum of 1.0 AMA PRA Category 1 Credit(s) ${ }^{\mathrm{TM}}$. Physicians should claim only the credit commensurate with the extent of their participation in the activity.

\footnotetext{
From the aivision of Hematology/Oncology, Laura and Isaac Perlmutter Cancer Center, New York University Langone Medical Center, and DDivision of Solid Tumor Oncology, Gastrointestinal Oncology Service, Department of Medicine, Memorial Sloan Kettering Cancer Center, and Weill College of Medicine, Cornell University, New York, New York. Submitted May 15, 2014; accepted for publication September 26, 2014. Dr. Giuroiu has disclosed that she has no financial interests, arrangements, affiliations, or commercial interests with the manufacturers of any products discussed in this article or their competitors. Dr. Reidy-Lagunes has disclosed that she is on the advisory board for Novartis AG, Pfizer Inc., and Ipsen. She also receives research support from Novartis AG.

Correspondence: Diane Reidy-Lagunes, MD, MS, Division of Solid Tumor Oncology, Gastrointestinal Oncology Service, Department of Medicine, Memorial Sloan Kettering Cancer Center, 300 East 66th Street, New York, NY 10128. E-mail: reidyd@mskcc.org
}

NCCN is accredited as a provider of continuing nursing education by the American Nurses Credentialing Center's Commission on Accreditation.

This activity is accredited for 1.0 contact hours. Accreditation as a provider refers to recognition of educational activities only; accredited status does not imply endorsement by NCCN or ANCC of any commercial products discussed/displayed in conjunction with the educational activity. Kristina M. Gregory, RN, MSN, OCN, is our nurse planner for this educational activity.

All clinicians completing this activity will be issued a certificate of participation. To participate in this journal CE activity: 1) review the learning objectives and author disclosures; 2) study the education content; 3 ) take the posttest with a $66 \%$ minimum passing score and complete the evaluation at http://education.nccn.org/ node/61223; and 4) view/print certificate.

Release date: February 18, 2015; Expiration date: February 18, 2016

\section{Learning Objectives}

Upon completion of this activity, participants will be able to:

- Assess the various treatment options available for patients with insulin secreting metastatic panNETs including regional therapy, systemic cytotoxic therapy, and systemic targeted therapy

- Discuss the challenges of symptom management in patients with metastatic insulinoma

EDITOR

Kerrin M. Green, MA, Assistant Managing Editor, JNCCN-Journal of the National Comprehensive Cancer Network

Ms. Green has disclosed that she has no relevant financial relationships.

\section{CE AUTHORS}

Deborah J. Moonan, RN, BSN, Director, Continuing Education, has disclosed that she has no relevant financial relationships.

Ann Gianola, MA, Manager, Continuing Education Accreditation \& Program Operations, has disclosed that she has no relevant financial relationships.

Kristina M. Gregory, RN, MSN, OCN, Vice President, Clinical Information Operations, has disclosed that she has no relevant financial relationships. Rashmi Kumar, PhD, Senior Manager, Clinical Content, has disclosed that she has no relevant financial relationships.

Deborah A. Freedman-Cass, PhD, Oncology Scientist/Senior Medical Writer, has disclosed that she has no relevant financial relationships. 
Well-differentiated neuroendocrine tumors (NETs) are rare neoplasms arising from the diffuse neuroendocrine cell system. Pancreatic NETs (panNETs), formerly called islet cell carcinomas, develop from the endocrine tissues of the pancreas (ie, islets of Langerhans) and have a 5-year overall survival rate of approximately $50 \%$ when metastasis is present at diagnosis. ${ }^{1}$ Tumor grade, as defined by Ki- 67 proliferation index, influences overall survival, with low-grade tumors portending a better outcome than intermediate- and high-grade tumors. ${ }^{2}$ panNETs pose a significant challenge because of tumor heterogeneity, varying degrees of aggressiveness, and diverse clinical presentations. For example, patients with low-grade, low-volume, nonhormone secreting tumors may be completely asymptomatic and, in the authors' opinion, should be observed first to monitor disease pace. Therapy should be initiated at disease progression and/or development of symptoms. An understanding of the symptoms and tumor biology is critical to individualize management of these uncommon tumors.

Functional panNETs, including insulinomas, gastrinomas, glucagonomas, and vasoactive intestinal polypeptide (VIPomas), secrete a variety of hormones, which lead to different clinical syndromes. ${ }^{3-5}$ These syndromes can often be effectively managed with somatostatin analogues (ie, octreotide or lanreotide). However, over time, most tumors will progress despite the use of somatostatin analogue therapy.

This report discusses a patient with a metastatic insulinoma whose multidisciplinary treatment included both regional and systemic therapies. Unfortunately, the patient succumbed to his disease 10 years after the original diagnosis.

\section{Case Report}

A 60-year-old man initially presented with right upper quadrant pain that he had been experiencing for approximately 5 to 6 months. In November 2003, an abdominal ultrasound revealed a pancreatic mass and hepatic lesions, and a CT scan showed hypodensities in both lobes of the liver. Results of fine-needle aspiration of a liver lesion were positive for metastatic well-differentiated panNET. No Ki-67 proliferation index or grade was reported because of the small amount of material obtained. The tumors $(>12$ and bilobar, with the largest being $10.1 \times 6.1 \mathrm{~cm}$ ) were deemed unresectable and medical management was recommended. At that time, the patient had no symptoms suggestive of a hormone-secreting tumor, and therefore no hormone markers were drawn.

In March 2004, because of the extensive nature of the disease, the patient began chemotherapy with capecitabine and temozolomide, and serial CT scans showed that the tumors began to decrease in size. $\mathrm{He}$ tolerated chemotherapy extremely well with few side effects, and the doses were never reduced. The patient remained on therapy for 3 years until his hepatic lesions were observed to increase slightly. The regimen was discontinued, and the patient remained clinically stable and continued to work full-time. In late 2007, he began to feel weakness after exercising and dizziness in the early morning. Insulin and proinsulin levels were elevated (insulin: $38 \mathrm{mcIU} / \mathrm{mL}$, upper limit of normal [ULN], $17 \mathrm{mcIU} / \mathrm{mL}$; proinsulin: $232 \mathrm{pmol} / \mathrm{L}$, ULN, $12 \mathrm{pmol} / \mathrm{L}$ ). Results of an octreotide scan were positive for uptake in the liver but not the pancreas, and treatment with $20 \mathrm{mg}$ of long-acting release octreotide via a monthly intramuscular injection was initiated in April 2008. His hypoglycemic symptoms, although initially controlled, became more severe and frequent. He experienced a syncopal episode at work in June 2008, and diazoxide and dexamethasone were added but provided little relief. The patient remained asymptomatic for only a few weeks before hypoglycemic episodes recurred. He underwent hepatic arterial bland embolization, with a resultant decrease in the size of the dominant hepatic masses and temporary resolution of his symptoms.

In November 2008, the patient was enrolled in a phase II study of MK-0646, an anti-insulinlike growth factor-1 receptor (IGF-R1) antibody, in patients with metastatic NETs (ClinicalTrials.gov identifier: NCT00610129). He tolerated therapy well, and imaging was stable according to RECIST criteria for 5 months. However, he experienced clinical disease progression with recurrent episodes of hypoglycemia, and required urgent hepatic arterial embolization. $\mathrm{He}$ remained asymptomatic until imaging results revealed disease progression in October 2009.

He was then enrolled in a phase II trial of bevacizumab and temsirolimus (ClinicalTrials.gov identifier: NCT01010126). However, his treatment course was complicated by grade 3 mucositis, grade 2 thrombocytopenia, and grade 2 fatigue, which led to disenrollment from the trial in March 2010 despite a dose reduction. In August 2010, the patient again 
became hypoglycemic and CT scan results showed disease progression. He was then briefly enrolled in a trial with the Akt inhibitor MK-2206 (ClinicalTrials.gov identifier: NCT01169649), but experienced rapid disease progression, and then underwent a brief course of sunitinib treatment. However, he was admitted for multiple episodes of hypoglycemia and underwent 2 more rounds of hepatic arterial embolization. The patient was then treated with everolimus from December 2010 to February 2011, and again experienced rapid disease progression. He underwent another round of hepatic arterial embolization, with a resultant radiographic response, but remained symptomatic with low blood glucose levels despite a high dose of dexamethasone and diazoxide. He then began a course of treatment with streptozocin, doxorubicin, and fluorouracil (5-FU) in April 2011, with no response requiring an increase in the dose of dexamethasone to control his symptoms.

In August 2011, 8 years after his original diagnosis, the cancer continued to progress and the patient was found comatose at his home twice, with severe hypoglycemic shock; both times he recovered only after his wife administered glucagon injections. After another round of hepatic artery embolization, the patient began treatment with gemcitabine, 1000 $\mathrm{mg} / \mathrm{m}^{2}$ and oxaliplatin, $80 \mathrm{mg} / \mathrm{m}^{2}$ every 2 weeks (GEMOX). In October 2011, because of fatigue, doses were reduced to $650 \mathrm{mg} / \mathrm{m}^{2}$ of gemcitabine and 60 $\mathrm{mg} / \mathrm{m}^{2}$ of oxaliplatin, which was then further reduced to $50 \mathrm{mg} / \mathrm{m}^{2}$. That same month, imaging results showed a notable response to treatment with this regimen, with a decrease in the size of all lesions and involved lymph nodes. However, by January 2012 (5 months after starting GEMOX), the patient developed symptomatic hyperglycemia. Measurement of glucagon levels confirmed the absence of a glucagonoma, and he was started on oral hypoglycemic therapy with sitagliptin. At this time, he also developed mild neuropathy. The frequency of chemotherapy dosing was decreased, and insulin was administered before each chemotherapy dose.

The patient remained on this therapy until December 2012, receiving a total of 17 months of gemcitabine/platinum therapy, with a confirmed partial response. His tumor progressed in January 2013. He had a brief treatment break until August 2013, when he was initiated on platinum/etoposide therapy with disease progression, and was eventually placed on best supportive care. He passed away peacefully in December 2013, 10 years after his original diagnosis.

\section{Discussion}

Insulinomas are exceedingly rare, with an estimated incidence reported to be 4 cases in 1 million person-years, and the 5-year survival rate can be up to $56 \%{ }^{6,7}$ Data from the SEER registry show a median age at diagnosis of 59 years, with a range spanning from childhood to more than 80 years of age. ${ }^{5}$ Fewer than 1 in 100,000 insulinomas are malignant, with malignancy defined by the presence of metastases. ${ }^{7,8}$ Germline DNA testing for hereditary tumor syndromes is recommended in patients with a familial history or clinical findings suggesting multiple endocrine neoplasia type 1 , tuberous sclerosis, or von Hippel-Lindau disease; the presence of multiple tumors; or the presence of precursor lesions in the peritumoral pancreatic tissue. ${ }^{9}$

Patients with insulinomas can present with a range of symptoms associated with hypoglycemia, especially experienced while fasting or exercising. ${ }^{4}$ These include manifestations of sympathetic overdrive (eg, weakness, diaphoresis, tremors, palpitations, hyperphagia) and neuroglycopenia (eg, confusion, visual changes, altered consciousness, convulsions, amnesia or coma). ${ }^{4}$ Symptom progression during a 72-hour fast in the setting of increased serum C-peptide levels and undetectable sulfonylurea levels remains the gold standard for diagnosis of an insulinoma. ${ }^{4}$

Treatment of insulinomas follows the same paradigm as that of other panNETs, and requires treating the hormone-related symptoms and the tumor. Although surgery remains the definitive treatment modality for tumors localized to the pancreas, ${ }^{4}$ several chemotherapeutic and targeted molecular agents have shown varying degrees of efficacy in treating metastatic disease.

Octreotide and diazoxide have been used primarily for symptom relief but, unfortunately, have transient benefit. ${ }^{4,10}$ Octreotide has been shown to control hypoglycemia efficiently in $35 \%$ to $50 \%$ of patients. ${ }^{4}$ However, before initiating octreotidebased therapy, an octreotide scan (ie, somatostatin analogue scintigraphy) should be performed to confirm somatostatin receptor expression in vivo., ${ }^{9,11}$ Administration of somatostatin to a patient whose 
tumor does not bind octreotide (ie, Octreoscan-negative tumors) may lead to a blunting of the compensatory glucagon response and may actually worsen hypoglycemia. ${ }^{9,12}$ Diazoxide is also sometimes used to control hypoglycemia; however, it can cause edema and hirsutism, and often the disease becomes refractory. ${ }^{12}$ Nighttime dextrose infusions to manage hypoglycemia can be used as an alternative to diazoxide and dexamethasone. Additionally, dietary counseling on the intake of complex carbohydrates also can help limit the frequency and severity of hypoglycemic episodes.

\section{Regional Therapy Treatment}

Selective bland embolization, chemoembolization, and radioembolization (using yttrium 90 beads) are frequently used as a palliative technique in patients with symptomatic hepatic metastases who are not candidates for surgical resection. ${ }^{13,14}$ Duration of response is highly variable, ranging from 4 months to 2 years, reflecting the differences in tumor biology. ${ }^{15-20}$ No prospective randomized trials have compared the clinical efficacy of these embolization methods. Patient selection is important to minimize treatmentrelated adverse effects, which can include pain, nausea, fever, fatigue, and liver abnormalities.

\section{Systemic Cytotoxic Therapy Treatment}

No clearly defined role currently exists for conventional chemotherapy in the treatment of metastatic panNETs. Most clinicians support the use of cytotoxic chemotherapy, such as streptozocin, 5-FU, platinum, or temozolomide, in patients with a high tumor burden and symptoms and/or disease progression. However, which cytotoxic chemotherapies should be used and in what order is currently unclear (eg, it is unknown whether a patient treated with temozolomide-based therapy would benefit from streptozocin on progression, and vice versa). No prospective randomized data exist for a temozolomide-based regimen. However, a single-institution series showed promising activity at 70\% (21 of 30 patients), based on RECIST criteria, with median progression-free survival (PFS) of 18 months. ${ }^{21}$ Reported response rates to streptozocin plus doxorubicin or 5-FU range from $16 \%$ to $69 \%$. $3,22,23$

\section{Systemic Targeted Therapy Treatment}

PI3K/Akt/mTOR Pathway: Targeted therapy against multiple steps in the IGF-R1-activated $\mathrm{PI} 3 \mathrm{~K} / \mathrm{Akt} / \mathrm{mTOR}$ pathway has shown various de- grees of response in NETs. The RADIANT-3 study was a randomized phase III study evaluating the efficacy of everolimus in advanced panNETs. ${ }^{24}$ Median PFS was 11.0 months for everolimus compared with 4.6 months for placebo (hazard ratio [HR], 0.35; 95\% CI, $0.27-0.45 ; P<.001$ ), which led to FDA approval of everolimus for this indication.

IGF-R1 showed promise as a target for downregulating mTOR signaling in preclinical trials. ${ }^{25} \mathrm{How}$ ever, a phase II trial of the anti-IGF-R1 monoclonal antibody MK-0646 failed to show an objective response, with a median PFS of 4.2 months in patients with advanced panNETs. ${ }^{26}$

It is thought that rapamycin analogues such as everolimus may specifically inhibit CREB-regulated transcription coactivator 1 (CRTC1), decreasing feedback inhibition on IGF-R1, and consequently upregulating Akt activity. ${ }^{27}$ The authors conducted a phase II study of MK-2206, an orally active, allosteric Akt inhibitor, which was terminated early based on a business decision by the sponsor. On study, 8 patients were treated (6 carcinoid, 2 panNET) and no complete or partial responses were seen. However, 1 patient (with an atypical thymic carcinoid tumor with brain metastases refractory to all standard therapies, including mTOR and vascular endothelial growth factor [VEGF] inhibitors) experienced a 17\% decrease in tumor size (SD) for more than 10 months. ${ }^{28}$

VEGF Pathway: The high vascularity of NETs lends itself to trials of targeted therapy against proangiogenic molecules. ${ }^{29}$ Sunitinib malate, a monoclonal antibody against VEGF receptors 2 and 3, plateletderived growth factor receptors (PDGFRs) $\alpha$ and $\beta$, and stem-cell factor receptor (c-kit), has been shown to slow tumor growth. ${ }^{30}$ Median PFS reported in a phase III randomized, double-blind, placebocontrolled trial was 11.4 months in the sunitinib arm compared with 5.5 months with placebo (HR, 0.42; 95\% CI, 0.26-0.66; $P<.001$ ), which led to FDA approval of sunitinib for the treatment of advanced panNETs. ${ }^{30}$

A phase II trial combining the anti-VEGF monoclonal antibody bevacizumab and the mTOR inhibitor temsirolimus showed a response rate of $41 \%$ and a PFS rate of $79 \%$ at 6 months. ${ }^{31}$ Further investigation is ongoing.

Radiolabeled Somatostatin Analogue Therapy: Radionuclide somatostatin analogue therapy, also known as peptide receptor radiotherapy, is offered in 
Europe for patients with advanced indium octreotidepositive NETs. This therapy is not yet available in the United States outside of clinical trials, and therefore was not offered to this patient. Several radioisotopes, linked to a somatostatin analogue, have been used, including indium-111 (111In), yttrium-90 (90Y), and lutetium-177 (177Lu). Studies of the 90Y-labeled somatostatin analogue, a highenergy $\beta$-particle emitter, reported response rates of up to $27 \% .^{32,33}$ An analysis of 504 patients with metastatic NETs receiving $177 \mathrm{Lu}$-octreotate reported a response rate of approximately 30\%.34,35

Ultimately, combination therapy with GEMOX was the most efficient regimen for sustainably controlling the present patient's hypoglycemia for more than 23 months, despite radiographic evidence of tumor growth. In a phase II study of gemcitabine monotherapy in patients with metastatic NETs, $65 \%$ of those enrolled originally achieved disease stabilization, but partial or complete responses were not noted in this group. ${ }^{36}$ A retrospective study of GEMOX therapy in 20 patients with advanced NETs who received at least 2 regimens of chemotherapy showed a $17 \%$ response rate and a median PFS of 7 months. ${ }^{37}$

\section{Conclusions}

The present patient's treatment course highlights the efficacy of both systemic cytotoxic chemotherapy and targeted therapies in a disease that was traditionally thought to be chemotherapy-resistant. Platinum-based therapy controlled his advanced and refractory disease for up to 23 months. This response may reflect the tumor's evolution over time. Regional therapy with hepatic artery embolization was also successful in controlling symptoms, but targeted therapies were not as successful. Both mTOR and VEGF inhibitors were administered after disease progression on temozolomide-based therapies. Whether cytotoxic therapy is contributing to the development of oncogenic transformation is unclear, but this could be a plausible explanation. Further investigation is needed to better define proper sequencing of these therapies. In summary, this case report describes the varying therapies that can be used to control tumor burden and maintain a patient's quality of life.

\section{References}

1. Strosberg JR, Cheema A, Weber J, et al. Prognostic validity of a novel American Joint Committee on Cancer Staging Classification for pancreatic neuroendocrine tumors. J Clin Oncol 2011;29:3044-3049.

2. Yao JC, Hassan M, Phan A, et al. One hundred years after "carcinoid": epidemiology of and prognostic factors for neuroendocrine tumors in 35,825 cases in the United States. J Clin Oncol 2008;26:3063-3072.

3. Rivera E, Ajani JA. Doxorubicin, streptozocin, and 5-fluorouracil chemotherapy for patients with metastatic islet-cell carcinoma. Am J Clin Oncol 1998;21:3638.

4. Metz DC, Jensen RT. Gastrointestinal neuroendocrine tumors: pancreatic endocrine tumors. Gastroenterology 2008;135:1469-1492.

5. Yao JC, Eisner MP, Leary C, et al. Population-based study of islet cell carcinoma. Ann Surg Oncol 2007;14:3492-3500.

6. Service FJ, McMahon MM, O’Brien PC, Ballard DJ. Functioning insulinomaincidence, recurrence, and long-term survival of patients: a 60-year study. Mayo Clin Proc 1991;66:711-719.

7. Strosberg J, Gardner N, Kvols L. Survival and prognostic factor analysis in patients with metastatic pancreatic endocrine carcinomas. Pancreas 2009;38:255-258.

8. Roland CL, Bian A, Mansour JC, et al. Survival impact of malignant pancreatic neuroendocrine and islet cell neoplasm phenotypes. J Surg Oncol 2012;105:595-600.

9. Vezzosi D, Bennet A, Rochaix P, et al. Octreotide in insulinoma patients: efficacy on hypoglycemia, relationships with Octreoscan scintigraphy and immunostaining with anti-sst2A and anti-sst5 antibodies. Eur J Endocrinol 2005;152:757-767.

10. Theodoropoulou M, Stalla GK. Somatostatin receptors: from signaling to clinical practice. Front Neuroendocrinol 2013;34:228-252.

11. Reidy DL, Tang LH, Saltz LB. Treatment of advanced disease in patients with well-differentiated neuroendocrine tumors. Nat Clin Pract Oncol 2009;6:143152.

12. Jensen RT, Cadiot G, Brandi ML, et al; Barcelona Consensus Conference participants. ENETS Consensus Guidelines for the management of patients with digestive neuroendocrine neoplasms: functional pancreatic endocrine tumor syndromes. Neuroendocrinology 2012;95:98-119.

13. Ruszniewski P, Rougier P, Roche A, et al. Hepatic arterial chemoembolization in patients with liver metastases of endocrine tumors. A prospective phase II study in 24 patients. Cancer 1993; 71:2624-2630.

14. Eriksson B, Larsson EG, Skogseid BM, et al. Liver embolizations of patients with malignant neuroendocrine gastrointestinal tumors. Cancer 1998;83:22932301.

15. Gupta S, Yao J, Ahrar K, et al. Hepatic artery embolization and chemoembolization for treatment of patients with metastatic carcinoid tumors: the MD Anderson Experience. Cancer 2003;9:261-267.

16. Moertel C, Johnson $C$, McKusick M, et al. The management of patients with advanced carcinoid tumors and islet cell carcinomas. Ann Intern Med 1994;120:302-309.

17. Kennedy AS, Dezarn WA, McNeillie P, et al. Radioembolization for unresectable neuroendocrine hepatic metastases using resin 90Y-microspheres: early results in 148 patients. Am J Clin Oncol 2008;31:271-279.

18. Rhee TK, Lewandowski RJ, Liu DM, et al. 90 Y radioembolization for metastatic neuroendocrine liver tumors: preliminary results from a multi-institutional experience. Ann Surg 2008;247:1029-1035.

19. King J, Quinn R, Glenn DM, et al. Radioembolization with selective internal radiation microspheres for neuroendocrine liver metastases. Cancer 2008;113:921-929.

20. Cao CQ, Yan TD, Bester L, et al. Radioembolization with yttrium microspheres for neuroendocrine tumour liver metastases. Br J Surg 2010;97:537-543.

21. Strosberg JR, Fine RL, Choi J, et al. First-line chemotherapy with capecitabine and temozolomide in patients with metastatic pancreatic endocrine carcinomas. Cancer 2011;117:268-275.

22. Moertel CG, Lefkopoulo M, Lipsitz S, et al. Streptozocin-doxorubicin, streptozocin-fluorouracil or chlorozotocin in the treatment of advanced isletcell carcinoma. N Engl J Med 1992;326:519-523.

23. Cheng P, Saltz L. Failure to confirm major objective antitumor activity for streptozocin and doxorubicin in the treatment of patients with advanced islet cell carcinoma. Cancer 1999;86:944-948.

24. Yao JC, Shah MH, Ito $T$, et al. Everolimus for advanced pancreatic neuroendocrine tumors. N Engl J Med 2011;364:514-523.

25. von Wichert G, Haeussler U, Greten FR, et al. Regulation of cyclin D1 expression by autocrine IGF-I in human BON neuroendocrine tumour cells. Oncogene 2005;24:1284-1289. 
26. Reidy-Lagunes DL, Vakiani E, Segal MF, et al. A phase 2 study of the insulinlike growth factor-1 receptor inhibitor MK-0646 in patients with metastatic, well-differentiated neuroendocrine tumors. Cancer 2012;118:4795-4800.

27. Chandarlapaty S, Sawai A, Scaltriti M, et al. AKT inhibition relieves feedback suppression of receptor tyrosine kinase expression and activity. Cancer Cell 2011;19:58-71.

28. Reidy-Lagunes D, Pietanza MC, Segal M, et al. A phase II clinical and translational study of MK-2206 in patients with metastatic neuroendocrine tumors (NETs). Presented at the 6th Annual Meeting of the North American NeuroEndocrine Tumor Society (NANETS); October 4-5, 2013; Charleston, NC.

29. Oberg K, Casanovas O, Castano JP, et al. Molecular pathogenesis of neuroendocrine tumors: implications for current and future therapeutic approaches. Clin Cancer Res 2013;19:2842-2849.

30. Raymond E, Dahan L, Raoul JL, et al. Sunitinib malate for the treatment of pancreatic neuroendocrine tumors [published correction appears in N Engl J Med 2011;364:1082]. N Engl J Med 2011;364:501-513.

31. Hobday TJ, Qin R,Reidy-Lagunes D, et al. Multicenter phase II trial of temsirolimus and bevacizumab in pancreatic neuroendocrine tumors. J Clin Oncol 2014, in press.
32. Walderr C, Pless M, Maecke HR, et al. The clinical value of [90Y-DOTA] dPhe-Tyr-octreotide in the treatment of neuroendocrine tumours: a clinical phase II study. Ann Oncol 2001;12:941-945.

33. Waldherr $\mathrm{C}$, Pless $\mathrm{M}$, Maecke $\mathrm{H}$, et al. Tumor response and clinical benefit in neuroendocrine tumors after $7.4 \mathrm{GBq}$ (90)Y-DOTATOC. J Nucl Med 2002;43:610-616.

34. Kwekkeboom DJ, de Herder WW, Kam BL, et al. Treatment with the radiolabeled somatostatin analog [177 Lu-DOTA 0,Tyr3] octreotate: toxicity, efficacy, and survival. J Clin Oncol 2008;26:2124-2130.

35. Kwekkeboom DJ, Teunissen JJ, Bakker WH, et al. Treatment with the radiolabeled somatostatin analogue [177Lu-DOTA0,Tyr3]octreotate in patients with gastroenteropancreatic tumors. J Clin Oncol 2005;23:2754-2762.

36. Kulke MH, Kim H, Clark JW, et al. A phase II trial of gemcitabine for metastatic neuroendocrine tumors. Cancer 2004;101:934-939.

37. Cassier PA, Walter T, Eymard B, et al. Gemcitabine and oxaliplatin combination chemotherapy for metastatic well-differentiated neuroendocrine carcinomas: a singlecenter experience. Cancer 2009;115:3392-3399.

\section{Instructions for Completion}

To participate in this journal CE activity: 1) review the learning objectives and author disclosures; 2 ) study the education content; 3 ) take the posttest with a $66 \%$ minimum passing score and complete the evaluation at http://education.nccn.org/ node/61223; and 4) view/print certificate. After reading the article, you should be able to answer the following multiple- choice questions. Credit cannot be obtained for tests completed on paper. You must be a registered user on NCCN.org. If you are not registered on NCCN.org, click on "New Member? Sign up here" link on the left hand side of the Web site to register. Only one answer is correct for each question. Once you successfully answer all posttest questions you will be able to view and/or print your certificate. Software requirements: Internet.

\section{Posttest Questions}

1. True or False: Syndromes associated with functional panNETs can often be effectively managed with somatostatin analogues (ie, octreotide or lanreotide).

2. Which of the following regional therapies are used in the palliative treatment of patients with metastatic panNETs and symptomatic liver lesions?

a. Radioembolization

b. Chemoembolization

\section{c. Bland embolization \\ d. All of the above}

3. Which 2 targeted agents are approved for progressive unresectable and/or metastatic pancreatic NETs?

a. Everolimus and sunitinib

b. Sorafenib and temsirolimus

c. Sorafenib and sunitinib

d. Everolimus and bevacizumab

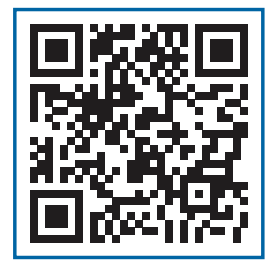

